\title{
Cardiovascular disease and 1,5-anhydro-d-glucitol
}

\author{
Nobutaka Ikeda ${ }^{1, *}$, Yukio Hiroi ${ }^{2}$ \\ ${ }^{1}$ Division of Cardiovascular Medicine, Toho University Ohashi Medical Center, Tokyo, Japan; \\ ${ }^{2}$ Department of Cardiology, National Center for Global Health and Medicine, Tokyo, Japan.
}

\begin{abstract}
The serum 1,5-anhydro-d-glucitol (1,5-AG) level rapidly decreases concomitantly with urinary glucose excretion in hyperglycemia. 1,5-AG is a sensitive clinical marker of short-term glycemic control, postprandial hyperglycemia and glucose fluctuation. Increasing evidence about the relationship between cardiovascular disease (CVD) and glucose fluctuations have been published. In this review, we summarize the possibilities and limitations of 1,5-AG as a marker of CVD. Research showed that 1,5-AG level is associated with prevalence of CVD and is also a predictive value for cardiovascular (CV) events. Especially in a high risk population, the predictive value of 1,5-AG for $\mathrm{CV}$ events becomes more effective. Besides, 1,5-AG is an effective glycometabolic marker that complements HbA1c in terms of glucose fluctuation. Appropriate use of 1,5-AG might lead to improved prognosis for patients or decrease medical financial burden of the population through early detection of glucose disorder and quality glucose control.
\end{abstract}

Keywords: Cardiovascular disease, 1,5-anhydro-d-glucitol, biomarker, prognosis

\section{Introduction}

Type 2 diabetes mellitus (DM) extremely impairs the prognosis of patients with cardiovascular disease (CVD) (1). Currently, because of the well-established relationships between micro-vascular disease and hemoglobin A1c (HbA1c), the American Diabetes Association (ADA) recommends the evaluation of $\mathrm{HbA} 1 \mathrm{c}$ as a criterion for diagnosing DM (2). The United Kingdom Prospective Diabetes Study (UKPDS) and the Diabetes Control and Complications Trial (DCCT) have shown that long-term favorable glycemic control improves the complications of DM $(3,4)$. However, in patients with advanced DM, HbA1c guided intensive glucose control does not always reduce macro-vascular complications, and in some cases increases the risk of death (5-7). To improve the patient's prognosis, early diagnosis and early intervention for glycemic abnormalities are essential (4). Increasing evidence has reported that postprandial hyperglycemia and glucose level fluctuation impairs mortality rate and promotes CVD progression (8-14). Feasible and sensitive clinical markers to detect glucose fluctuations are needed.

\section{1,5-anhydro-d-glucitol (1,5-AG)}

1,5-AG is a monosaccharide originating primarily from dietary sources that is found in constant concentrations in the blood in normal glycemic status (15). 1,5-AG is a naturally occurring 1-deoxy form of glucose (Figure
1) and was discovered in a milkwort plant, Polygala senega in 1888. Further investigation was repeated and became commercially available in Japan from 1991 (Table 1). Approximately 500 to $1,000 \mathrm{mg} 1,5-\mathrm{AG}$ exists in the human body mainly in its free form (15). 1,5-AG is adjusted by urinary excretion in the kidneys. Most $1,5-\mathrm{AG}$, which is filtered in glomerulus is reabsorbed at a specific fructose-mannose active transporter in the renal tubule $(15,16)$ (Figure 2A). The reabsorption is competitively inhibited by glucose. Therefore, the serum 1,5-AG level rapidly decreases when the serum glucose level exceeds the threshold of urine glucose excretion $(160-180 \mathrm{mg} / \mathrm{dL})$ and is an important and feasible clinical marker of short-term glycemic status (Figure 2B) $(17,18)$. Therefore, low $1,5-\mathrm{AG}$ is associated with postprandial hyperglycemia or poor glycemic control status. Yamanouchi reported distribution of 1,5-AG levels in Japanese healthy subjects (Male: $26.6 \pm 7.2 \mu \mathrm{g}$ / mL, Female: $21.5 \pm 6.0 \mu \mathrm{g} / \mathrm{mL}$, Total: $24.6 \pm 7.2 \mu \mathrm{g} / \mathrm{mL}$ ) (19). There is no significant statistical difference between lower limits of healthy males and females (Male: 14.2 $\mu \mathrm{g} / \mathrm{mL}$, Female: $13.5 \mu \mathrm{g} / \mathrm{mL}$ ). Therefore, as the normal lower limit of 1,5-AG levels, a cut-off value of $14.0 \mu \mathrm{g} /$ $\mathrm{mL}$ calculated by all healthy subjects is recommended (19). The mean 1,5-AG value of patients with DM was $7.3 \pm 7.1 \mu \mathrm{g} / \mathrm{mL}$ and was significantly lower than that of healthy subjects (Figure 3) (19).

Unlike $\mathrm{HbA1c}$, although the 1,5-AG value is not affected by red blood cell life span, several clinical conditions should be considered in interpretation of 

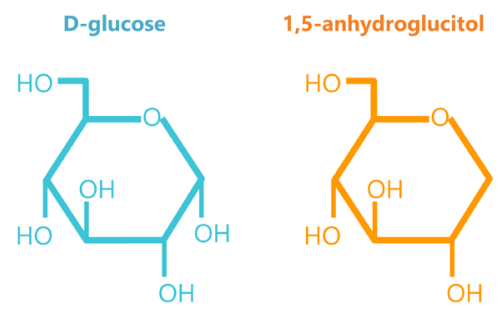

Figure 1. Molecular structures of D-glucose and 1,5-Anhydro-d-glucitol (1,5-AG).
Table 1. History of 1,5-Anhydro-d-glucitol

\begin{tabular}{ll}
\hline Year & Items \\
\hline 1888 & $\begin{array}{l}\text { 1,5-AG discovered in a milkwort plant, Polygala senega } \\
\text { Low levels discovered in patients with diabetes by Pitkänen } \\
\text { (Finland) }\end{array}$ \\
1975 & $\begin{array}{l}\text { Studied the relationship between diabetes and 1,5-AG levels } \\
\text { in Japan }\end{array}$ \\
1987 & $\begin{array}{l}\text { Developed kits measuring 1,5-AG by Nippon Kayaku } \\
\text { Commercially available in Japan from 1991 }\end{array}$ \\
20031 & $\begin{array}{l}\text { US FDA approval (Bland name is GLYCOMARK) } \\
\text { CE Marked }\end{array}$ \\
\hline
\end{tabular}

(A)

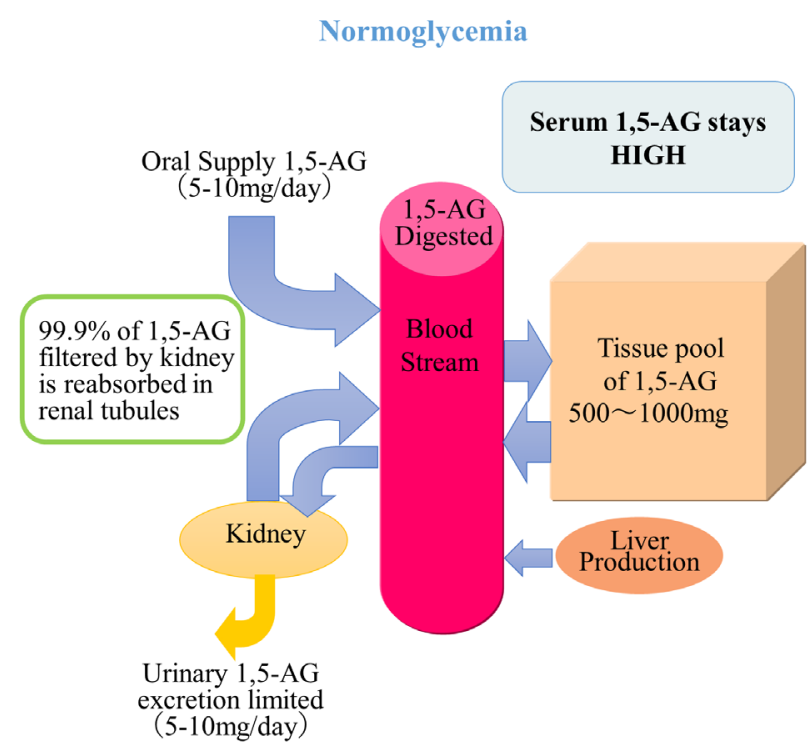

(B)

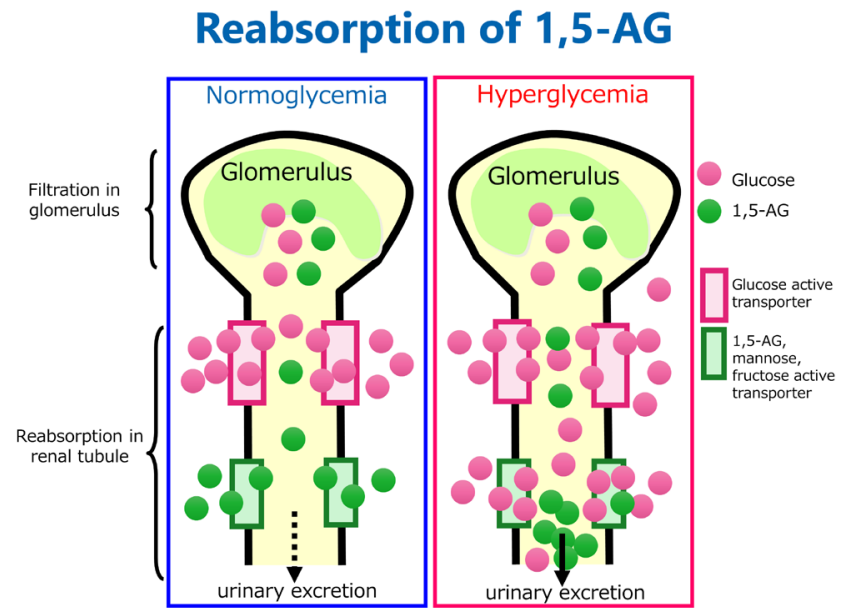

- Like glucose, 1,5-AG is filtered out of the bloodstream and transported back into the bloodstream.

- Primary source of $1,5-\mathrm{AG}$ is food, though up to $10 \%$ is synthesized in liver.

- 1,5-AG does not metabolized much in body.

- Under normal glucose condition, 1,5-AG levels in blood are stable, with balance between ingestion and excretion supported by large body pool of 1,5-AG. Thus, 1,5-AG levels in blood are not affected by meal.

- When glucose levels exceed the renal threshold (hyperglycemia excursions), glucose is excreted in the urine (glycosuria).

- Due to the similarities in molecules, large amount of $1,5-\mathrm{AG}$ is also excreted during glycosuria.

- 1,5-AG serum levels fall rapidly, representing hyperglycemia during the prior 1-2 weeks.

- The greater the duration, magnitude and frequent hyperglycemia, the lower 1,5-AG levels.

Figure 2. Metabolism of 1,5-Anhydro-dglucitol (1,5-AG). (A) In vivo kinetics of 1,5AG; (B) Filtration and reabsorption of 1,5AG in the kidney. Normoglycemia: Nearly $99.9 \%$ of filtered $1,5-\mathrm{AG}$ at the glomerulus is reabsorbed in the renal tubule. Hyperglycemia (over 160-180 mg/dL): Competitive tubular reabsorption of glucose and 1,5-AG.
1,5-AG values (Table 2). Recently the use of sodiumglucose cotransporter 2 inhibitor (SGLT2i) has been rapidly increasing. Although SGLT2i improves glycemic control, serum 1,5-AG decreases. The reabsorption of 1,5-AG occurs predominantly via a SGLT4 and the effect of SGLT2i for 1,5-AG reabsorption is indirect (20). The amount of glycosuria caused by SGLT2i is about 60$80 \mathrm{~g} /$ day. Serum 1,5-AG level will be reduced to $0-1 \mu \mathrm{g} /$ $\mathrm{mL}$ in a week, when a large amount of glycosuria (above $50 \mathrm{~g} /$ day) appears. If SGLT2 $\mathrm{i}$ therapy causes substantial glycosuria, the serum 1,5-AG level will generally decrease to near $1 \mu \mathrm{g} / \mathrm{mL}$ in a week after administration. If serum 1,5-AG levels increase during administration, it could show decreasing effectiveness of SGLT2i.

\section{Postprandial hyperglycemia and glucose fluctuation}

It is well known that postprandial hyperglycemia is associated with cardiovascular (CV) events (14). Recently, there has been increasing evidence that fluctuations in glucose levels leads to endothelial dysfunction and increases the risk of $\mathrm{CV}$ events and the progression of 


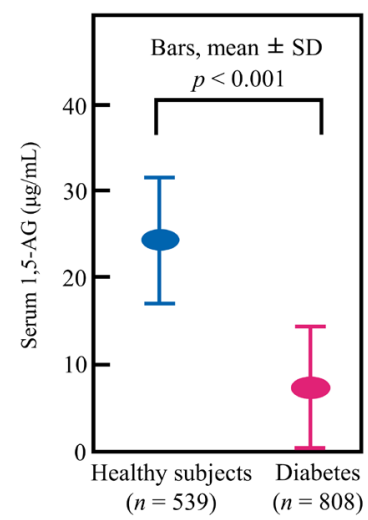

Figure 3. Serum 1,5-AG levels of healthy subjects and patients with diabetes mellitus. (Modified from Yamanouchi T, et al. Journal of the Japan Diabetes Society. 1990; 33:4147. $($ Ref. 19))

Table 2. Clinical factors affecting 1,5-Anhydro-d-glucitol

\begin{tabular}{ll}
\hline Increased & Decreased \\
\hline Chinese herbal drugs containing & Females \\
Polygalae Radix & Newborns \\
Some enteral nutrition products & Renal failure \\
& Glomerulonephritis \\
& Pregnancy \\
& Total parenteral nutrition \\
& Acarbose \\
& Sodium-glucose cotransporter 2 \\
& inhibitor (SGLT2i) \\
\hline
\end{tabular}

atherosclerosis $(21,22)$. Glucose fluctuations have a bad effect on coronary plaque vulnerability and progression in patients with CAD (10-13). These findings may explain the increased $\mathrm{CV}$ mortality rate during intensive glycemic therapy. Most evidence about glucose fluctuation and $\mathrm{CV}$ events or coronary plaques are based on continuous glucose monitoring (CGM) and oral glucose tolerance tests (OGTT) (10-14). 1,5-AG can be used to differentiate patients with glucose fluctuation despite having a similar HbA1c (23).

As a reduced 1,5-AG value strongly correlates with glucose excursions, it is also associated with postprandial hyperglycemia $(17,18)$. Furthermore, 1,5-AG values have been correlated with the mean amplitude of glycemic excursions (MAGE) and other parameters of CGM $(23,24)$. Therefore, $1,5-\mathrm{AG}$ has potential to be a marker for CVD and a predictor of CV events.

\section{Relationship with CVD and events}

Prevalence of coronary artery disease, carotid artery disease and acute ischemic stroke/transient ischemic attack

Several previous reports demonstrated that $1,5-\mathrm{AG}$ is associated with prevalence of coronary artery disease (CAD) and carotid artery disease (25-28). Patients with
CAD showed significantly lower 1,5-AG values than those without CAD $(25,28)$. Fujiwara presented that serum 1,5-AG was significantly lower in patients with CAD $(16.6 \pm 8.50 v s .21 .1 \pm 7.97 \mu \mathrm{g} / \mathrm{mL}, p<0.001)$ (28). Ikeda showed that patients with CAD presented significantly lower 1,5-AG and higher HbA1c values than patients without CAD (11.6 vs. $17.6 \mu \mathrm{g} / \mathrm{mL}, p$ $<0.001$, and $6.0 \%$ vs. $5.7 \%, p<0.001$, respectively) (25). In addition, 1,5-AG values have a correlation with severity of CAD assessed by SYNTAX score $(\rho=-0.27$, $p<0.001)(25)$. Even in patents without DM, serum $1,5-\mathrm{AG}$ values can be a help to detect the prevalence of $\mathrm{CAD}$, and the correlation is superior to $\operatorname{HbA1c}(26,28)$. Watanabe evaluated carotid arteries of 72 patients without DM and CVD by high-resolution ultrasonography. They reported that a higher pulsatility index of carotid arteries is associated with lower 1,5-AG values, but intima media thickness was not correlated with 1,5-AG values (27). They suggested that 1,5-AG is associated with stiffness but not with morphological changes of carotid arteries (27). Shiga demonstrated that a low serum 1,5-AG value $(1,5-\mathrm{AG}<14 \mu \mathrm{g} / \mathrm{mL})$ is a marker for acute ischemic stroke or transient ischemic attacks in patients with wellcontrolled DM (HbA1c < 7\%) (29).

\section{Prediction of cardiovascular events}

There is a certain consensus that $1,5-\mathrm{AG}$ can be used to predict cardiovascular events in patients with DM. The evidence was reported that a lower serum 1,5-AG value is associated with cardiovascular events, mainly from Japan (30-35) (Table 3).

Watanabe showed that the measurement of serum 1,5$\mathrm{AG}$ is useful to detect high risk men for CVD, regardless of the presence or absence of diabetes (34). This report is a relatively long-term (11 years) population-based cohort study. The usefulness of 1,5-AG was limited. Selvin demonstrated the data from Atherosclerosis Risk in a Communities (ARIC) study. Compared with persons with $1,5-\mathrm{AG} \geq 6.0 \mu \mathrm{g} / \mathrm{mL}$ and no history of $\mathrm{DM}$, persons with $\mathrm{DM}$ and $1,5-\mathrm{AG}<6.0 \mu \mathrm{g} / \mathrm{mL}$ showed an increased risk of cardiovascular events (35). However, the predictive value of CVD or CV events was inadequate in the population without diagnosis of DM (35). The study subjects of these two cohort studies were low risk for CVD or a healthy population $(34,35)$. In such a low risk population, the effectiveness of 1,5-AG for prediction of $\mathrm{CV}$ events might not have been fully evaluated. On the other hand, in high risk populations, the predictive value of 1,5-AG for $\mathrm{CV}$ events is excellent. Low serum $1,5-\mathrm{AG}$ values are significantly associated with cardiac mortality or adverse clinical events in patients with CAD (31-33). Fujiwara evaluated 141 patients after percutaneous coronary intervention (PCI) with followup coronary angiography (CAG). Median 1,5-AG values were significantly lower in patients with coronary revascularization (13.4 vs. $18.7 \mu \mathrm{g} / \mathrm{mL}, p<0.01, p=$ 
Table 3. Publication of 1,5-Anhydro-d-glucitol for prediction of cardiovascular events

\begin{tabular}{|c|c|c|c|c|}
\hline First author (ref.) & Year & Patients Number & Location & Main Finding \\
\hline Ikeda N (30) & 2016 & 889 & Japan & $\begin{array}{l}\text { Low } 1,5-A G \text { value predicts cardiac and cerebrovascular events even in non-DM patients } \\
\text { without CAD. Cut off value of } 1,5-\mathrm{AG} \text { is } 10 \mu \mathrm{g} / \mathrm{mL} \text {. }\end{array}$ \\
\hline Takahashi S (32) & 2016 & 200 & Japan & $\begin{array}{l}\text { Low } 1,5-\mathrm{AG} \text { value is associated with adverse clinical events in patients with } \mathrm{HbA} 1 \mathrm{c}< \\
7.0 \% \text { after first time elective percutaneous coronary intervention }\end{array}$ \\
\hline Selvin E (35) & 2016 & 11,106 & USA & $\begin{array}{l}\text { Compared with persons with } 1,5-\mathrm{AG} \geq 6.0 \mu \mathrm{g} / \mathrm{mL} \text { and no history of } \mathrm{DM} \text {, persons with } \\
\mathrm{DM} \text { and } 1,5-\mathrm{AG}<6.0 \mu \mathrm{g} / \mathrm{mL} \text { showed increased risk of cardiovascular events. Participants } \\
\text { in the Atherosclerosis Risk in Communities (ARIC) study without CVD at baseline }\end{array}$ \\
\hline Ouchi S (33) & 2017 & 388 & Japan & $\begin{array}{l}\text { Low } 1,5-\mathrm{AG} \text { value predicted long-term cardiac mortality in patients with acute coronary } \\
\text { syndrome and } \mathrm{HbA} 1 \mathrm{c}<7.0 \% \text {. }\end{array}$ \\
\hline Shiga Y (29) & 2017 & 1,246 & Japan & $\begin{array}{l}\text { Low serum } 1,5-\mathrm{AG} \text { value is a marker for acute ischemic stroke or transient ischemic } \\
\text { attacks in patients with well-controlled DM. }\end{array}$ \\
\hline Watanabe M (34) & 2011 & 2,095 & Japan & $\begin{array}{l}\text { The measurement of serum 1,5-AG value is useful to detect high risk men for CVD, } \\
\text { regardless of the presence or absence of diabetes. }\end{array}$ \\
\hline Fujiwara T (31) & 2016 & 141 & Japan & $\begin{array}{l}\text { Lower } 1,5-\mathrm{AG} \text { is a risk factor for adverse clinical events after percutaneous coronary } \\
\text { intervention. }\end{array}$ \\
\hline
\end{tabular}

$\mathrm{CAD}$, coronary artery disease; CVD, cardiovascular disease.

0.005) (31). Takahashi showed that a low 1,5-AG value was associated with adverse clinical events after first time elective PCI even in patients with well-controlled DM (HbA1c < 7.0\%) (32). Ouchi presented that low 1,5AG levels predict long-term cardiac mortality in patients with acute coronary syndrome with HbA1c levels < $7.0 \%$. The 1,5-AG value of the cardiac death group was significantly lower than that of the survivor group $(12.3 \pm 5.3$ vs. $19.2 \pm 7.7 \mu \mathrm{g} / \mathrm{mL}, p<0.01)(33)$. Ikeda reported that in a high risk population, a low 1,5-AG value predicts major cardiac and cerebrovascular events (MACCE) even in non-DM patients without CAD. The study subjects were the patients who needed their first CAG. Therefore, these patients were potentially high risk even if CAG did not reveal CAD. The low 1,5-AG group $(1,5-\mathrm{AG}<10.0 \mathrm{mg} / \mathrm{mL})$ showed significantly higher risk of not only MACCE but also all causes of death (30).

\section{Conclusions}

1,5-AG level is associated with prevalence of CV disease and has also predictive value for CV events. Especially in a high risk population, the predictive value for $\mathrm{CV}$ events of 1,5-AG becomes more effective. Measurement of serum 1,5-AG values is useful for not only evaluation of individual glucose control but also population risk assessment from a public health perspective. 1,5-AG is an effective glycometabolic marker that complements $\mathrm{HbA} 1 \mathrm{c}$ in terms of glucose fluctuation. Appropriate use of 1,5-AG might lead to improved prognosis for patients or decreased medical financial burden of the population through early detection of glucose disorders and quality glucose control.

\section{References}

1. Kannel WB, McGee DL. Diabetes and cardiovascular disease. The Framingham study. JAMA. 1979; 241:20352038.

2. American Diabetes Association. Diagnosis and classification of diabetes mellitus. Diabetes Care. 2010; 33 Suppl 1:S62-S69.

3. Writing Team for the Diabetes Control and Complications Trial/Epidemiology of Diabetes Interventions and Complications Research Group. Effect of intensive therapy on the microvascular complications of type 1 diabetes mellitus. JAMA. 2002; 287:2563-2569.

4. Holman RR, Paul SK, Bethel MA, Matthews DR, Neil HA. 10-year follow-up of intensive glucose control in type 2 diabetes. N Engl J Med. 2008; 359:1577-1589.

5. Action to Control Cardiovascular Risk in Diabetes Study Group, Gerstein HC, Miller ME, Byington RP, Goff DC Jr, Bigger JT, Buse JB, Cushman WC, Genuth S, IsmailBeigi F, Grimm RH Jr, Probstfield JL, Simons-Morton DG, Friedewald WT. Effects of intensive glucose lowering in type 2 diabetes. N Engl J Med. 2008; 358:2545-2559.

6. ADVANCE Collaborative Group, Patel A, MacMahon $\mathrm{S}$, et al. Intensive blood glucose control and vascular outcomes in patients with type 2 diabetes. N Engl J Med. 2008; 358:2560-2572.

7. Duckworth W, Abraira C, Moritz T, et al. Glucose control and vascular complications in veterans with type 2 diabetes. N Engl J Med. 2009; 360:129-139.

8. Bonora E, Muggeo M. Postprandial blood glucose as a risk factor for cardiovascular disease in Type II diabetes: the epidemiological evidence. Diabetologia. 2001; 44:21072114.

9. Ceriello A. The possible role of postprandial hyperglycaemia in the pathogenesis of diabetic complications. Diabetologia. 2003; 46 Suppl 1:M9-M16.

10. Kuroda M, Shinke T, Sakaguchi K, Otake H, Takaya T, Hirota Y, Osue T, Kinutani H, Konishi A, Takahashi H, Terashita D, Uzu K, Hirata K. Association between daily glucose fluctuation and coronary plaque properties in patients receiving adequate lipid-lowering therapy assessed by continuous glucose monitoring and optical coherence tomography. Cardiovasc Diabetol. 2015; 14:78.

11. Kuroda M, Shinke T, Sakaguchi K, et al. Effect of daily glucose fluctuation on coronary plaque vulnerability 
in patients pre-treated with lipid-lowering therapy: a prospective observational study. JACC Cardiovasc Interv. 2015; 8:800-811.

12. Gohbara M, Hibi K, Mitsuhashi T, Maejima N, Iwahashi N, Kataoka S, Akiyama E, Tsukahara K, Kosuge M, Ebina T, Umemura S, Kimura K. Glycemic variability on continuous glucose monitoring system correlates with nonculprit vessel coronary plaque vulnerability in patients with first-episode acute coronary syndrome-optical coherence tomography study. Circ J. 2016; 80:202-210.

13. Kataoka S, Gohbara M, Iwahashi N, Sakamaki K, Nakachi T, Akiyama E, Maejima N, Tsukahara K, Hibi K, Kosuge M, Ebina T, Umemura S, Kimura K. Glycemic variability on continuous glucose monitoring system predicts rapid progression of non-culprit lesions in patients with acute coronary syndrome. Circ J. 2015; 79:2246-2254.

14. Glucose tolerance and mortality: comparison of WHO and American Diabetes Association diagnostic criteria. The DECODE study group. European Diabetes Epidemiology Group. Diabetes Epidemiology: Collaborative analysis Of Diagnostic criteria in Europe. Lancet. 1999; 354:617-621.

15. Yamanouchi $\mathrm{T}$, Tachibana $\mathrm{Y}$, Akanuma H, Minoda S, Shinohara T, Moromizato H, Miyashita H, Akaoka I. Origin and disposal of 1,5-anhydroglucitol, a major polyol in the human body. Am J Physiol. 1992; 263(2 Pt 1):E268-E273.

16. Yamanouchi T, Shinohara T, Ogata N, Tachibana Y, Akaoka I, Miyashita H. Common reabsorption system of 1,5-anhydro-D-glucitol, fructose, and mannose in rat renal tubule. Biochim Biophys Acta. 1996; 1291:89-95.

17. Yamanouchi T, Akanuma Y. Serum 1,5-anhydroglucitol (1,5 AG): new clinical marker for glycemic control. Diabetes Res Clin Pract. 1994; 24 Suppl:S261-S268.

18. Dungan KM. 1,5-anhydroglucitol (GlycoMark) as a marker of short-term glycemic control and glycemic excursions. Expert Rev Mol Diagn. 2008; 8:9-19.

19. Yamanouchi T, Akanuma Y, Toyota T, Kuzuya T, Kawai T, Kawazu S, Yoshioka S, Kanazawa Y, Ohta M, Baba S, Kosaka K. Clinical significance of serum 1,5-anhydroglucitol measurements in diabetes mellitus. Journal of the Japan Diabetes Society. 1990; 33:41-47. (in Japanese)

20. Tazawa S, Yamato T, Fujikura H, Hiratochi M, Itoh F, Tomae M, Takemura Y, Maruyama H, Sugiyama T, Wakamatsu A, Isogai T, Isaji M. SLC5A9/SGLT4, a new $\mathrm{Na}+$-dependent glucose transporter, is an essential transporter for mannose, 1,5-anhydro-D-glucitol, and fructose. Life Sci. 2005; 76:1039-1050.

21. Chen XM, Zhang Y, Shen XP, Huang Q, Ma H, Huang YL, Zhang WQ, Wu HJ. Correlation between glucose fluctuations and carotid intima-media thickness in type 2 diabetes. Diabetes Res Clin Pract. 2010; 90:95-99.

22. Monnier L, Mas E, Ginet C, Michel F, Villon L, Cristol JP, Colette C. Activation of oxidative stress by acute glucose fluctuations compared with sustained chronic hyperglycemia in patients with type 2 diabetes. JAMA. 2006; 295:1681-1687.

23. Dungan KM, Buse JB, Largay J, Kelly MM, Button EA, Kato S, Wittlin S. 1,5-anhydroglucitol and postprandial hyperglycemia as measured by continuous glucose monitoring system in moderately controlled patients with diabetes. Diabetes Care. 2006; 29:1214-1219.

24. Sun J, Dou JT, Wang XL, Yang GQ, Lü ZH, Zheng $\mathrm{H}$, Ma FL, Lu JM, Mu YM. Correlation between 1,5-anhydroglucitol and glycemic excursions in type 2 diabetic patients. Chin Med J (Engl). 2011; 124:36413645.

25. Ikeda N, Hara H, Hiroi Y. 1,5-Anhydro-d-glucitol predicts coronary artery disease prevalence and complexity. J Cardiol. 2014; 64:297-301.

26. Ikeda N, Hara H, Hiroi Y. Ability of 1,5-Anhydro-d-glucitol values to predict coronary artery disease in a non-diabetic population. Int Heart J. 2015; 56:587-591.

27. Watanabe K, Suzuki T, Ouchi M, Suzuki K, Ohara M, Hashimoto M, Yamashita H, Okazaki M, Ishii K, Oba K. Relationship between postprandial glucose level and carotid artery stiffness in patients without diabetes or cardiovascular disease. BMC Cardiovasc Disord. 2013; 13:11.

28. Fujiwara T, Yoshida M, Yamada H, Tsukui T, Nakamura T, Sakakura K, Wada H, Arao K, Katayama T, Funayama H, Sugawara Y, Mitsuhashi T, Kakei M, Momomura S, Ako J. Lower 1,5-anhydroglucitol is associated with denovo coronary artery disease in patients at high cardiovascular risk. Heart Vessels. 2015; 30:469-476.

29. Shiga Y, Kuriyama M, Kanaya Y, Takeshima S, Takemaru M, Takamatsu K, Shimoe Y, Fujikawa Y, Nishigaki M. Serum 1,5-Anhydroglucitol: Risk factor of acute ischemic stroke and transient ischemic attack in well-controlled diabetes. Cerebrovasc Dis. 2017; 44:325-329.

30. Ikeda N, Hara H, Hiroi Y, Nakamura M. Impact of serum 1,5-anhydro-d-glucitol level on prediction of major adverse cardiac and cerebrovascular events in non-diabetic patients without coronary artery disease. Atherosclerosis. 2016; 253:1-6.

31. Fujiwara T, Yoshida M, Akashi N, et al. Lower 1,5-anhydroglucitol is associated with adverse clinical events after percutaneous coronary intervention. Heart Vessels. 2016; 31:855-862.

32. Takahashi S, Shimada K, Miyauchi K, et al. Low and exacerbated levels of 1,5-anhydroglucitol are associated with cardiovascular events in patients after first-time elective percutaneous coronary intervention. Cardiovasc Diabetol. 2016; 15:145.

33. Ouchi S, Shimada K, Miyazaki T, et al. Low 1,5-anhydroglucitol levels are associated with long-term cardiac mortality in acute coronary syndrome patients with hemoglobin A1c levels less than 7.0. Cardiovasc Diabetol. 2017; 16:151.

34. Watanabe M, Kokubo Y, Higashiyama A, Ono Y, Miyamoto Y, Okamura T. Serum 1,5-anhydro-D-glucitol levels predict first-ever cardiovascular disease: An 11-year population-based cohort study in Japan, the Suita study. Atherosclerosis 2011; 216:477-483.

35. Selvin E, Rawlings A, Lutsey P, Maruthur N, Pankow JS, Steffes M, Coresh J. Association of 1,5-Anhydroglucitol with cardiovascular disease and mortality. Diabetes. 2016; 65:201-208.

Received September 15, 2019; Revised December 6, 2019.; Accepted December 15, 2019.

*Address correspondence to:

Nobutaka Ikeda, Division of Cardiovascular Medicine, Toho University Ohashi Medical Center, 2-22-36 Ohashi, Meguro-ku, Tokyo 153-8515, Japan.

E-mail: ikedanobutaka@yahoo.co.jp 